ALICE reference number ALICE-PUB-2000-29 version 1.0

Institute reference number

Date of last change 2001-03-14

\title{
Recent results from beam tests of large area silicon drift detectors
}

\author{
Authors: \\ E. Crescio, M. Bondila, V. Bonvicini, P. Cerello, P. Giubellino, A. Kolojvari, \\ M. I. Martinez, G. Mazza, L. M. Montano, D. Nouais, S. Piano, C. Piemonte, \\ A. Rashevsky, A. Rivetti, F. Tosello, A. Vacchi, R. Wheadon \\ for the ALICE Collaboration
}

\begin{abstract}
:
Silicon drift detectors with an active area of $7.0 \times 7.5 \mathrm{~cm}^{2}$ will equip the two middle layers of the Inner Tracking System of the ALICE experiment. The performance of several prototypes was studied during beam tests carried out at the CERN SPS facility. The results of the beam test data analysis are discussed in this paper.
\end{abstract}




\title{
Recent results from beam tests of large area silicon drift detectors
}

\author{
E. Crescio ${ }^{\mathrm{a}, 1}$, M. Bondila ${ }^{\mathrm{b}, 4}, \mathrm{~V}$. Bonvicini ${ }^{\mathrm{c}}$, P. Cerello $^{\mathrm{a}}$, \\ P. Giubellino ${ }^{\text {a }}$, A. Kolojvari ${ }^{\mathrm{a}, \mathrm{b}, 3}$, M. I. Martinez ${ }^{\mathrm{a}, 2}$, G. Mazza $^{\mathrm{a}}$, \\ L.M. Montaño ${ }^{\mathrm{a}, 2}$, D. Nouais ${ }^{\mathrm{a}}, \mathrm{S}$ Piano $^{\mathrm{c}}, \mathrm{C}$ Piemonte $^{\mathrm{c}}$, \\ A. Rashevsky ${ }^{c}$, A. Rivetti ${ }^{\text {a }}$, F. Tosello ${ }^{\text {a }}$, A. Vacchi ${ }^{c}$, \\ R. Wheadon ${ }^{\text {a }}$ for the ALICE Collaboration \\ ${ }^{a}$ Torino University and INFN-Sez.Torino, Italy \\ ${ }^{\mathrm{b}}$ Jyväskylä University, Finland \\ ${ }^{\mathrm{c}}$ INFN Sezione di Trieste, Italy
}

\begin{abstract}
Silicon drift detectors with an active area of $7.0 \times 7.5 \mathrm{~cm}^{2}$ will equip the two middle layers of the Inner Tracking System of the ALICE experiment. The performance of several prototypes was studied during beam tests carried out at the CERN SPS facility. The results of the beam test data analysis are discussed in this paper.
\end{abstract}

\section{Introduction}

Silicon Drift Detectors (SDDs) [1-3] are particle detectors well suited for high multiplicity experiments, since they can provide unambiguous two-dimensional position information and $d E / d x$ measurement with a reduced number of readout channels. In a SDD, under the effect of an applied electric field, the electrons generated by the crossing particle drift along a direction parallel to the surface of the wafer to an array of anodes perpendicular to the drift direction. The coordinate along the drift direction is determined by the measurement of the drift time, the second coordinate is obtained from the charge distribution

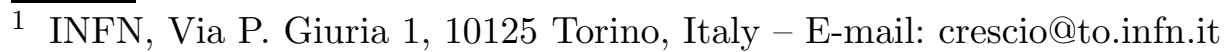

2 also at CINVESTAV Mexico City, Mexico

3 also at St.Petersburg University, St. Petersburg, Russia

4 on leave from Institute of Spatial Sciences, Bucharest, Romania 
across the anodes.

SDDs have been chosen to equip the two middle layers of the Inner Tracking System (ITS) of the ALICE experiment at the LHC [4]. The detectors will be produced on $3 \mathrm{k} \Omega \mathrm{cm} 5 " n$-type neutron transmutation doped silicon wafers, with an active area of $7.0 \times 7.5 \mathrm{~cm}^{2}$. The active region of each detector is divided centrally in two separate zones each with a $35 \mathrm{~mm}$ maximum drift path read out at the edge by 256 anodes with a pitch of $294 \mu \mathrm{m}$.

The results discussed in this paper were obtained from prototypes with characteristics close to those from the final design for ALICE [5], read out by the OLA preamplifier [6] and a Flash ADC system [7]. A brief description of the SDD front-end electronics designed for ALICE, which will be used for the first time in beam tests during this year, is also given.

\section{Beam test results}

The SDDs were exposed to the $375 \mathrm{GeV} / \mathrm{c}$ pion beam of the CERN SPS. They were placed on the beam trajectory between two microstrip telescopes which allow the measurement of the particle impact point on the SDD with a precision of $5 \mu m$ r.m.s..

Particular attention was devoted to the analysis of the detector spatial resolution, defined as the r.m.s. of the distribution of the residuals between the coordinates measured by the SDD and by the microstrip telescope.

In order to make this analysis, it is necessary to take into account the effects which lead to systematic errors in the determination of the coordinates along both directions, thus affecting the resolution.

For the resolution along the time direction, it is necessary to correct for the drift velocity variations due to temperature instability during time and to the temperature gradient present in the detector, caused by the integrated resistive voltage divider heating.

The position measurement is also affected by the presence of fluctuations in the doping concentration within the wafer [8]. These fluctuations cause a nonuniformity of the drift field, which alters the proportionality between the drift time and the distance of the crossing point of the particle from the anodes, and a parasitic electric field component along the anode direction, which induces deviations of the electron trajectories from the ideal linear path. In fig. 1 the values of the resolution along the drift direction as a function of the drift distance, before correction for the doping fluctuations, are shown. The tested detector presented a systematic non-linearity along the drift time axis, due to the punch-through current between cathodes. The systematic non-linearities were corrected with a polynomial fit. The black circles represent the result obtained studying a large area (40 anodes) of the detector. These values can be considered an upper limit of the drift time resolution. This preliminary 


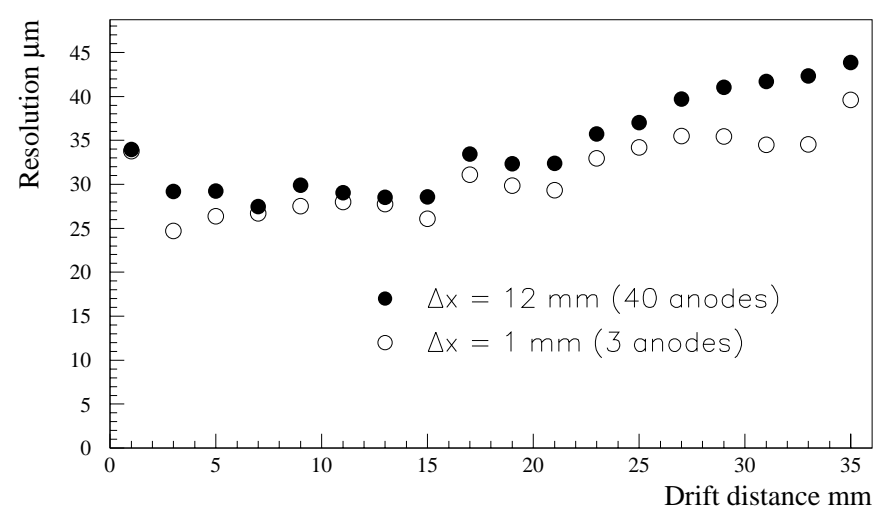

Fig. 1. Resolution along the drift direction as a function of the drift distance. The black circles represent the resolution obtained studying an area corresponding to 40 anodes, the light circles represent the values for an area corresponding to 3 anodes.

result was obtained taking into account the temperature effects but assuming a uniformly doped wafer. As shown in the figure (light circles), a selection of any small region $(1 \mathrm{~mm})$ along the anode direction, in which the doping concentration can be considered independent of the anodic coordinate, allows the improvement the resolution. This suggests that a global improvement can be obtained after the correction for the doping fluctuations.

In fig. 2 the curve with triangles shows the anodic resolution corrected for the doping fluctuations. In order to optimize the anodic resolution, it is also possible to correct the anodic coordinate (obtained from the centroid of the charge distribution across the anodes) by using a technique based on the good knowledge of the electron cloud evolution during the drift [9].

Usually, the anodic coordinate is determined by the center of gravity $\left(x_{C o G}\right)$ of the charge measured by the anodes. This value can be significantly different from the centroid of the electron cloud gaussian distribution. A correction function $\Delta x\left(x_{C o G}, \sigma\right)$ can be applied, where $\sigma$ represents the r.m.s. of the gaussian distribution and depends on the drift time and on the released charge. Neglecting in first approximation the Coulomb repulsion effect during the drift, the time dependence of the cluster size is $\sigma(t)=\sqrt{2 D t+\sigma_{0}^{2}}$, where $D$ is the diffusion coefficient and $\sigma_{0}$ represents the initial size of the cloud.

The black curve in fig. 2 illustrates the improvement obtained for the anodic resolution by applying such a CoG correction. The correction is applied only for the two-anode clusters. A global improvement of the resolution can be observed. The degradation in the region close to the anodes is due to the intrinsic position uncertainty of the single-anode clusters. 


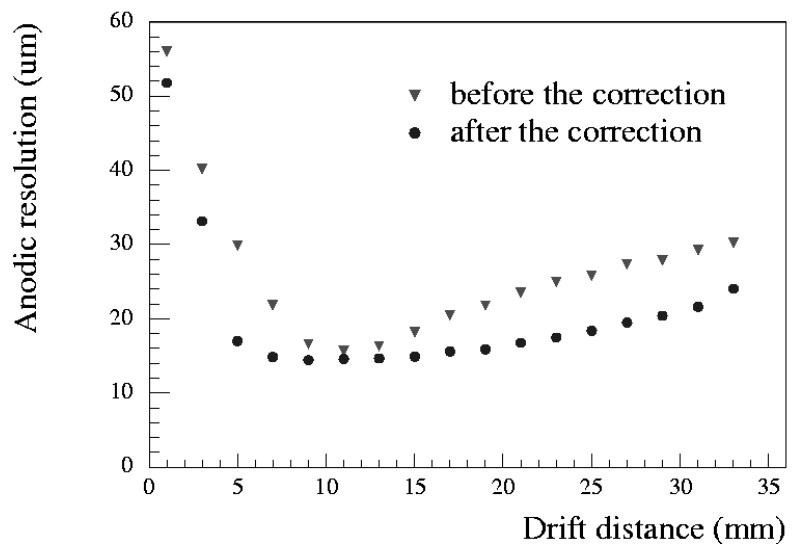

Fig. 2. Anodic resolution already corrected for the doping fluctuations along the anode direction as a function of the drift distance before and after the centroid correction.

\section{The ALICE front-end readout unit}

A schematic representation of the front-end readout unit designed for ALICE is shown in fig. 3. It is based on two ASICs, implemented with a $0.25 \mu \mathrm{m}$

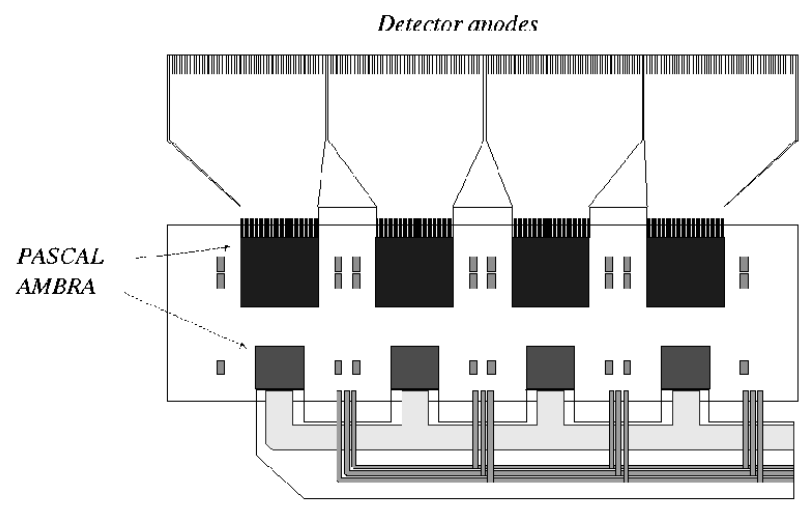

Fig. 3. The front-end readout unit for the ALICE silicon drift detectors.

CMOS radiation tolerant technology. The first one, PASCAL, performs low noise preamplification, analog storage and analog to digital conversion. The second one, AMBRA, is a multi-event digital buffer. Since the SDDs are very sensitive to temperature variation, the average power consumption should be kept below $5 \mathrm{~mW} /$ anode. The required sampling frequency, in order to improve the separation of close tracks, is $40 \mathrm{MHz}$. At this speed the design of an ADC with this low power consumption is very difficult. The adopted solution is to store continuously the data coming from the detector in a ring analog memory. The data are digitized and transferred to the DAQ only when the trigger system validates them. In this way the most power demanding components work for a reduced percentage of the time strongly reducing the power con- 
sumption. Prototypes of both ASICs have been produced: test results show that the circuits fulfil the ALICE requirements [10,11]. During the next beam tests, a 32-channel prototype of PASCAL will be used.

L. M. M and M. I. M wish to thank Conacyt for financial support.

\section{References}

[1] E. Gatti and P. Rehak, Nucl. Instr. and Meth. A225 (1984) 608-614.

[2] S. Beolé et al., Nucl. Instr. and Meth. A377 (1996) 393-396.

[3] S. Beolé et al., Il Nuovo Cimento Vol. 109A, N. 9 (1996).

[4] ALICE Collaboration, CERN/LHCC 99-12, http://alice.web.cern.ch/Alice/documents.html.

[5] A. Rashevsky et al., proceedings at 8th Pisa Meeting on advanced detector, La Biodola, Isola d'Elba, Italy, 21-27 May 2000.

[6] W. Dabrowski et al., Nucl. Phys. B 44 (1995) 637.

[7] F. Balestra et al., Nucl. Instr. and Meth. A323 (1992) 523.

[8] D. Nouais et al., proceedings at 8th Pisa Meeting on advanced detector, La Biodola, Isola d'Elba, Italy, 21-27 May 2000.

[9] E. Crescio, D. Nouais and P. Cerello, ALICE-INT-2001-09, http://alice.web.cern.ch/Alice/documents.html.

[10] A. Rivetti et al., accepted for publication in the TNS.

[11] G. Mazza et al., proceedings at LEB 2000, CERN 2000-010, CERN/LHCC/2000-041. 